\title{
Organización territorial y aprovechamiento turístico sustentable en el Parque Nacional Arrecifes de Cozumel, México
}

\author{
Territorial organization and sustainable tourist use in the \\ Cozumel Reefs National Park, México
}

\author{
Lucinda Arroyo Arcos ${ }^{1}$ \\ Romano Gino Segrado Pavón ${ }^{2}$ \\ Oscar Frausto Martínez ${ }^{3}$ \\ Cristopher Arturo González Baca ${ }^{4}$ \\ Orlando Colín Olivares ${ }^{5}$ \\ Universidad de Quintana Roo, México
}

\section{Resumen}

El espacio se declaró Área Natural Protegida (ANP) desde 1996 y en el año 2000, se determinó su recategorización como Parque Nacional Arrecifes de Cozumel (PNAC). Desde su creación ya existían servicios turísticos en su área de influencia, mismos que se han incrementado con el paso de los años. La metodología consistió en la revisión de literatura vinculada con el tema, realización

1 Dra. en Geografía por la Universidad Nacional Autónoma de México (UNAM), Profesora Investigadora de Carrera, Universidad de Quintana Roo, Unidad Académica Cozumel. Correo electrónico: larroyo@uqroo.edu.mx

2 Dr. en Ciencias Ambientales, Profesor Investigadora de Carrera, Universidad de Quintana Roo, Unidad Académica Cozumel. Correo electrónico: romano@uqroo.edu.mx

3 Dr. en Geografía por la Universidad Nacional Autónoma de México (UNAM), Profesor Investigador de Carrera, Universidad de Quintana Roo, Unidad Académica Cozumel. Correo electrónico: frausto@uqroo.edu.mx

4 Mtro. Ciencias Biológicas, Director de la Comisión Nacional de Áreas Protegidas (CONANP) de Cozumel. Correo electrónico: cristopher.gonzalezb@conanp.gob.mx

5 Egresado de la Maestría en Gestión Sustentable del Turismo de la Universidad de Quintana Roo, Unidad Académica Cozumel, Lic. En Ciencias Ambientales, líneas de investigación en gestión Integral de Playas y Manejo Integrado de la Zona Costera. Correo electrónico: colinolivareso@gmail.com

Este artículo corresponde a la ponencia presentada en el 35th Conference of Latin American Geographers realizada en San José, Costa Rica del 20 al 22 de mayo del 2018. 
de cinco talleres con permisionarios y cooperativistas, 12 entrevistas a informantes clave, cuatro recorridos en la zona de estudio y área de influencia para levantamiento de datos georreferenciados y llenado de fichas de observación. El objetivo fue revelar la organización territorial y el aprovechamiento turístico en el PNAC, entre los resultados se encontró una fuerte intensidad de las actividades turístico recreativas en las zonas arrecifales del PNAC, se destacan las fortalezas, oportunidades, debilidades y amenazas en el PNAC y como propuesta de organización del territorio, el establecimiento de unidades funcionales y nodos para el mejor aprovechamiento de los recursos en el ANP, se enfatiza una incompatibilidad entre la zonificación del programa de manejo vigente, con la concentración de las actividades turístico-recreativas que se practican en el área.

Palabras clave: Organización territorial, aprovechamiento turístico, Área Natural Protegida, Cozumel.

\begin{abstract}
In 1996, the space was declared Natural Protected Area (ANP) and in the year 2000, was determined as Cozumel Reefs National Park (PNAC). Since its creation, there were already tourist services in its area of influence, which have increased over the years. The methodology consisted in the review of literature related to the topic, five workshops with service providers and cooperative members, twelve interviews to key informants, four tours in the study zone and at the same time in the area of influence for georeferenced data lifting and filling observation sheets. The objective was to reveal the territorial organization and the tourist use in the PNAC. Among the results, was found a strong intensity of recreational tourist activities in the reef areas of the PNAC. The highlights are the strengths, opportunities, weakness, and threats in the PNAC. As proposal for the organization of the territory, the establishment of functional units and modes for the best use of resources in the ANP, is emphasized an incompatibility between the zoning of the management program in place, with the concentration of recreational tourist activities practiced in the area.
\end{abstract}

Keywords: Territorial organization, tourist use, natural protected area, Cozumel.

\title{
Introducción
}

El territorio tiene una nueva lectura y ha dejado de considerarse únicamente como soporte de las actividades económicas, y pasa a ser entendido como un recurso de múltiples dimensiones e interdependencias entre factores ambientales, económicos, sociales y culturales, como resultado de relaciones dinámicas entre naturaleza y la sociedad (García, 2003). Esta última se encuentra con el reto de construir nuevos espacios y modelos de desarrollo, ante el flujo intensivo de turistas en un mismo tiempo y espacio, lo que ha provocado la saturación de ciertos destinos con ello la masificación turística, por lo que se han implementado estrategias para diversificar la oferta a una modalidad de menor impacto en, espacios rurales, naturales y culturales, así surge el turismo alternativo (Sánchez y Cebrián, 2015).

La Secretaria de Turismo de México (SECTUR, 2004), para dirigir las actividades turísticas en aquellos territorios con cierta fragilidad, sugiere 
Lucinda Arroyo Arcos, Romano Gino Segrado Pavón, Oscar Frausto Martínez, Cristopher Arturo González Baca, Orlando Colín Olivares. Organización territorial y aprovechamiento turístico sustentable en el Parque Nacional

Arrecifes de Cozumel, México

el turismo de naturaleza y lo define como aquellos viajes donde se realizan actividades recreativas en contacto con los recursos naturales y expresiones culturales, bajo la modalidad de ecoturismo, turismo rural o turismo de aventura y quienes lo practican tienen el compromiso de conocer, respetar y conservar dichos recursos. Es común que la práctica del turismo de naturaleza se realice en Áreas Naturales Protegidas (ANP) que tienen su base en la Constitución, Ley General de Equilibrio Ecológico y Protección al Ambiente (LGEEPA) y el Reglamento de ANP, creadas mediante decreto presidencial, representan la conservación para asegurar el equilibrio y la continuidad de los procesos ecológicos, los valores culturales, paisajístico compatibles con un desarrollo sustentable (Comisión Nacional de Áreas Naturales Protegidas-CONANP, 2007), órgano desconcentrado de la Secretaria de Medio Ambiente y Recursos Naturales (SEMARNAT)

La planificación del territorio en Quintana Roo, se manifiesta a través de los Programas de Ordenamiento Ecológico Territorial (POET), en estos se establece la vocación del suelo en relación con las actividades económicas entre ellas la turística, la distribución territorial de estas actividades y de la población así como la zonificación del territorio y normas de aplicación y en cuanto a la LGEEPA en su Art. 46 los tipos y características de las Áreas Naturales Protegidas (ANP) en los destinos turísticos estatales y en sus artículos 47Bis, 47Bis 1, y 50 hacen referencia a las restricciones de uso y formas de aprovechamiento sustentable, en ANP como las actividades de investigación científica, educación ambiental, y de turísticas-recreativas de bajo impacto, así como la infraestructura de apoyo y aprovechamiento de los recursos naturales que satisfaga las necesidades de los pobladores (LGEEPA, 2012; Segrado, Arroyo y Amador, 2010).

Entre las ANP más importantes de Quintana Roo, se encuentra el Parque Nacional Arrecifes de Cozumel, decretado como Parque Marino en 1996 y recategorizado a PN en el año 2000, su mayor atractivo es su sistema arrecifal con una variedad de especies marinas, entre corales, gorgonáceos, peces, algas y esponjas. En el área de influencia a la zona protegida se encuentran servicios de usos turísticos, recursos o atractivos de uso público tales como: playas públicas, club de playas, restaurantes, hoteles, villas condominios o segundas residencias. Las actividades de mayor intensidad en la zona son el buceo, el esnórquel y la pesca deportiva-recreativa. 


\section{Objetivo general}

Revelar la organización territorial y el aprovechamiento turístico en el Parque Nacional Arrecifes de Cozumel (PNAC).

\section{Caracterización geográfica del área de estudio}

La isla de Cozumel está considerada como la tercera más grande del territorio mexicano, se ubica al este de la Península de Yucatán en las costas del estado Quintana Roo, su extensión territorial es de 48 kilómetros $(\mathrm{km})$ por $16 \mathrm{~km}$, colinda al norte y al sur con el municipio de Solidaridad y el Mar Caribe y al oeste con el municipio de Solidaridad. En esta isla se localiza el Parque Nacional Arrecifes de Cozumel (PNAC), con una extensión de $11,98787-50$ hectáreas y entre los $20^{\circ} 29^{\prime} 02.93$ " y $20^{\circ} 14^{\prime} 27.02^{\prime \prime}$ $\mathrm{N}$ y $86^{\circ} 53^{\prime} 11.54^{\prime \prime}$ y $87^{\circ} 03$ ' 32.07” W (INE, 1998). La superficie marino terrestre es de aproximadamente $120 \mathrm{~km}$, tiene una forma de herradura alrededor de la parte media baja de la isla de Cozumel e inicia en el arrecife Paraíso en la parte occidental y termina en Punta Chiqueros en la porción oriental (Decreto de creación PCAC-DOF, 1996) (Ver Ilustración 1). 
Lucinda Arroyo Arcos, Romano Gino Segrado Pavón, Oscar Frausto Martínez, Cristopher Arturo González Baca, Orlando Colín Olivares. Organización territorial y aprovechamiento turístico sustentable en el Parque Nacional Arrecifes de Cozumel, México

\section{Ilustración 1. Área de estudio: Parque Nacional Arrecifes de Cozumel}
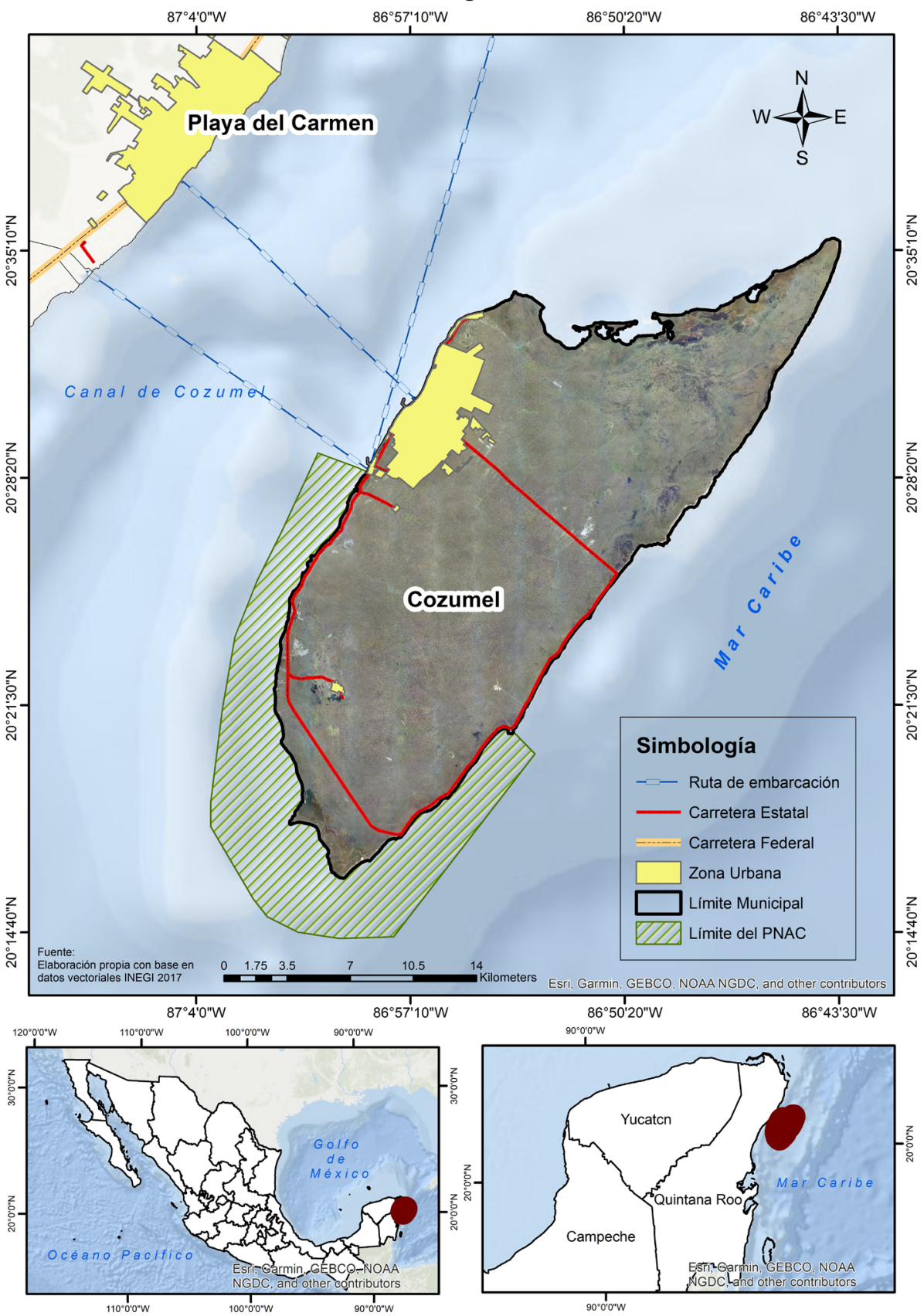

Fuente: elaboración propia con base en INEGI 2013, CONANP 2012. 
Una de las cuestiones importantes que forman parte de la organización del territorio es la parte estática que incluye el proceso histórico que junto a los elementos territoriales provocan cambio en los espacios. El antecedente de espacio protegido es desde el 11 junio 1980, que bajo Decreto Federal se crea una "Zona de Refugio para la Protección de la Flora y Fauna Marinas de la Costa Occidental de la Isla de Cozumel”. El 15 de julio de 1996 se publica un decreto el Diario Oficial del Gobierno del Estado de Quintana Roo, que otorga la categoría de Zona Sujeta a Conservación Ecológica, Refugio Estatal de Flora y Fauna, a la región denominada Laguna de Colombia, con una superficie total de 734.59 ha. Programa de Manejo (INE, 1998). El 19 de Julio de 1996, se decretó Parque Marino Nacional Arrecifes de Cozumel y el 7 de junio de 2000 con la modificación a la LGEEPA, se re-categorizó a Parque Nacional Arrecifes de Cozumel.

El ecosistema submarino del PNAC se integra por un sistema arrecifal de 988 especies marinas, con 459 géneros y 203 familias, los cuales incluyen 66 especies de corales duros, 39 gorgonáceos y 453 de peces, las algas representan 374 especies y las esponjas 56 y zonas de manglares hacia el litoral (Fenner, 1988; Reyes, 2007). En el PNAC se pueden encontrar especies en alguna categoría de protección de acuerdo con la NOM059-SEMARNAT-2010, como tortugas marinas (verde, caguama y carey) y los corales Acropora palmata y A. cervicornis, otras especies como el caracol rosado o blanco (Strombus gigas) y la langosta espinosa (Panulirus argus), entre otras (SEMARNAT, 2010, SEGOB, 2009).

\section{Marco teórico-conceptual}

En las investigaciones desde la geografía del turismo, destacan la variable espacial y territorial del hecho turístico para evidenciar la organización territorial Gómez, (2005), Vera (1997) y se recae en el análisis y la síntesis de sus diversos componentes como, el medio físico, actividades humanas, aspectos generales y concretos de la sociedad a través de las diversas interrelaciones que ocurren en el espacio (Almirón, 2004; Albet, 1993; Santos, 2000). Entre otras aportaciones se analizan el turismo y sus múltiples vinculaciones con el territorio y las transformaciones territoriales en donde la actividad de ocio se convierte en consumidora y productora de espacio geográfico (Pinassi y Ercolan, 2017, 2015; Hall, 2013). El territorio funge como el objeto de estudio bajo una estructura de acciones que se articulan con las diferentes escalas (local, 
Lucinda Arroyo Arcos, Romano Gino Segrado Pavón, Oscar Frausto Martínez, Cristopher Arturo González Baca, Orlando Colín Olivares. Organización territorial y aprovechamiento turístico sustentable en el Parque Nacional

Arrecifes de Cozumel, México

estatal y global) (Hiernaux y Lindón, 1993). Santos (1990), investiga el espacio geográfico como pieza clave, que no puede existir por sí sólo como algo distinto de lo tangible, es un conjunto de sistemas, que a su vez se desarrollan por medio de las funciones y las formas, a través de los procesos históricos presentes y pasados, que junto al ser humano se dan sobre la superficie terrestre y conforman el espacio como una estructura social.

Desde la perspectiva anterior se pueden destacar dos componentes: humano-naturaleza, que representan la realidad que conocemos, donde el primero transforma al medio físico que lo rodea y lo adapta para satisfacer sus necesidades, o bien interfiere directamente en la transformación y organización del territorio a través de los procesos y las estructuras (Delgadillo, Torres y Gasca, 2001 y Kostrowicki, 1986). Asimismo, se deja ver que el territorio se organiza en función de procesos y agentes internos y externos que tienden a marcar o delimitar el desarrollo de las actividades económicas e influyen en la orientación turística (Almirón, 2004).

Las investigaciones sobre turismo, territorio y ANP, indican que las actividades turístico-recreativas que se desarrollen en esos espacios deben ser de bajo impacto y compatibles con los objetivos de la gestión de área (Miranda y Alvarado, 2017, Dilschneider, 2016; Iradi, 2014); se discute que al favorecer la transformación de espacios naturales a turísticos se involucren intereses opuestos al uso y conservación (Briassoulis, 2002)., por lo que es necesario entrar una compatibilidad entre el turismo y el desarrollo sustentable en áreas protegidas, desde la filosofía del buen vivir como estrategia de procesos turísticos incluya a la comunidad (López, 2015; Vicencio y Bringas, 2014,), asimismo se plantean modelos para el aprovechamiento turístico sustentable en ANP (Segrado, Arroyo, Amador, Palma y Serrano, 2015; Segrado y Arroyo, 2009).

El aprovechamiento turístico sustentable se refiere a la utilización de los recursos naturales de tal forma que se respete su integridad funcional y la capacidad de carga de los ecosistemas por periodos definidos (LEGEEPA, 2012). Asimismo, favorece la competitividad de los destinos y el bienestar de las comunidades locales, para la conservación de los recursos son necesarias estrategias y normas, que regulen los límites para las actividades humanas y turísticas en espacios naturales o culturales (Segrado et al., 2014). La disponibilidad del espacio, la tecnología y los hábitos de consumo son determinantes del nivel de aprovechamiento para el turismo, y esto implica cuestionarse sobre cuáles son los límites que deben respetarse o priorizarse (Li y Lian, 2012). 
La actividad turística reconoce el modelo de aprovechamiento sustentable y lo vincula con la capacidad de carga turística, definida por la OMT (1981) como el número máximo de personas que pueden visitar un lugar al mismo tiempo, sin que causen destrucción en el medio físico, económico, socio-cultural. En México la Ley General de Turismo, reconoce que el aprovechamiento turístico sustentable conlleva el uso óptimo de los recursos naturales, culturales y valores tradicionales de las comunidades locales, con la finalidad de elevar su nivel de vida (Ley General de Turismo, 2009). Para el presente trabajo una de las herramientas que se considera dentro de la organización del territorio para el aprovechamiento turístico es, el programa de Manejo del Parque que incluye la zonificación para el uso del suelo estableciendo categorías de uso intensivo a bajo.

\section{Marco metodológico}

El PNAC existe una dinámica turística intensa tanto al interior como en su área de influencia, por lo que es necesario encontrar el equilibrio en su ámbito territorial, paisaje y en los umbrales, económicos, ecológicos, sociales y sobre todo en los objetivos de conservación y aprovechamiento sustentable de los recursos, planteados desde su creación por los gestores del ANP.

La investigación fue desarrolla en tres fases, la primera consistió en la revisión de literatura vinculada con el tema, así como de trabajos realizados sobre la zona de estudio, en la segunda fase se llevó a cabo el trabajo de campo para la recolección de información que consistió en cuatro recorridos a la zona de estudio: dos al área marítima con el apoyo del personal de la CONANP y dos en el área de influencia para el levantamiento de datos georreferenciados de la oferta turística y atractivos turísticos, además se levantaron fichas de observación.

Se diseño un guion de entrevista que incluyó preguntas para la detección de fortalezas, oportunidades, debilidades y amenazas, sobre políticas ambientales y de conservación, como maximizar el aprovechamiento turísticos en el ANP, detección de problemas ambientales, conflicto por posesión de tierras, entre otras, se hicieron 12 entrevistas a informantes clave: personal de la CONANP, representantes de asociaciones civiles, educativas, servidores públicos, del sector empresarial, dirección de ecológica, Fundación de Parques y Museos y representante de la zona marítimo terrestre. 
Parte fundamental en la recolección de datos fueron los cinco talleres con una participación de 72 permisionarios y cooperativistas conocedores del ANP (Ver tabla 1). La dinámica en la realización de talleres incluyó tres partes, la primera para explicar el objetivo del proyecto y lo que se esperaba con su participación, en la segunda se les pidió a los participantes llenar un instrumento diseñado para recolectar información de diagnóstico sobre las fortalezas, debilidades, oportunidades y amenazas sobre el aprovechamiento turístico en el ANP y en el tercer momento se utilizó la metodología de cartografía participativa para la detección de sitios de acceso, rutas de recorrido, problemáticas detectadas en el área sugerencias sobre un mejor aprovechamiento turístico en el PNAC.

Tabla 1 Talleres con permisionarios y cooperativistas

\begin{tabular}{|c|l|c|}
\hline \multicolumn{1}{|c|}{ Fecha } & \multicolumn{1}{|c|}{ Tipo de participante } & Total \\
\hline 29/sep/2014 & $\begin{array}{l}\text { Prestadores de servicio esnórquel y/o buceo en el PNAC } \\
\text { (permisionarios) }\end{array}$ & 11 \\
\hline $30 /$ sep/2014 & $\begin{array}{l}\text { Prestadores de servicio esnórquel y/o buceo en el PNAC } \\
\text { (permisionarios) }\end{array}$ & 12 \\
\hline 1/oct/2014 & Cooperativa turística Lagunas de Cozumel (cooperativistas) & 18 \\
\hline 2/oct/2014 & $\begin{array}{l}\text { Prestadores de servicio esnórquel y/o buceo en el PNAC } \\
\text { (permisionarios) }\end{array}$ & 15 \\
\hline $3 /$ oct/2014 & Cooperativa Pescadores de Cozumel (cooperativistas) & 16 \\
\hline & Total & 72 \\
\hline
\end{tabular}

Fuente: Elaboración propia.

\section{Resultados}

\section{Zonificación del PNAC}

La zonificación es el instrumento técnico de planeación utilizado en el establecimiento de las áreas naturales protegidas, que permite ordenar su territorio en función del grado de conservación y representatividad de sus ecosistemas, la vocación natural del terreno, de su uso actual y potencial, de conformidad con los objetivos dispuestos. Para determinar las unidades recreativas del PNAC, se hizo referencia al "Programa de Manejo Parque Marino Nacional Arrecifes de Cozumel, Quintana Roo", publicado en 1998 que presenta la zonificación de uso de suelo intensivo, restringido y de baja intensidad (Ilustración 2). 
Lucinda Arroyo Arcos, Romano Gino Segrado Pavón, Oscar Frausto Martínez, Cristopher Arturo González Baca,

Orlando Colin Olivares. Territorial organization and sustainable tourist use in the Cozumel Reefs National Park, México

\section{Ilustración 2. Zonificación del PNAC}

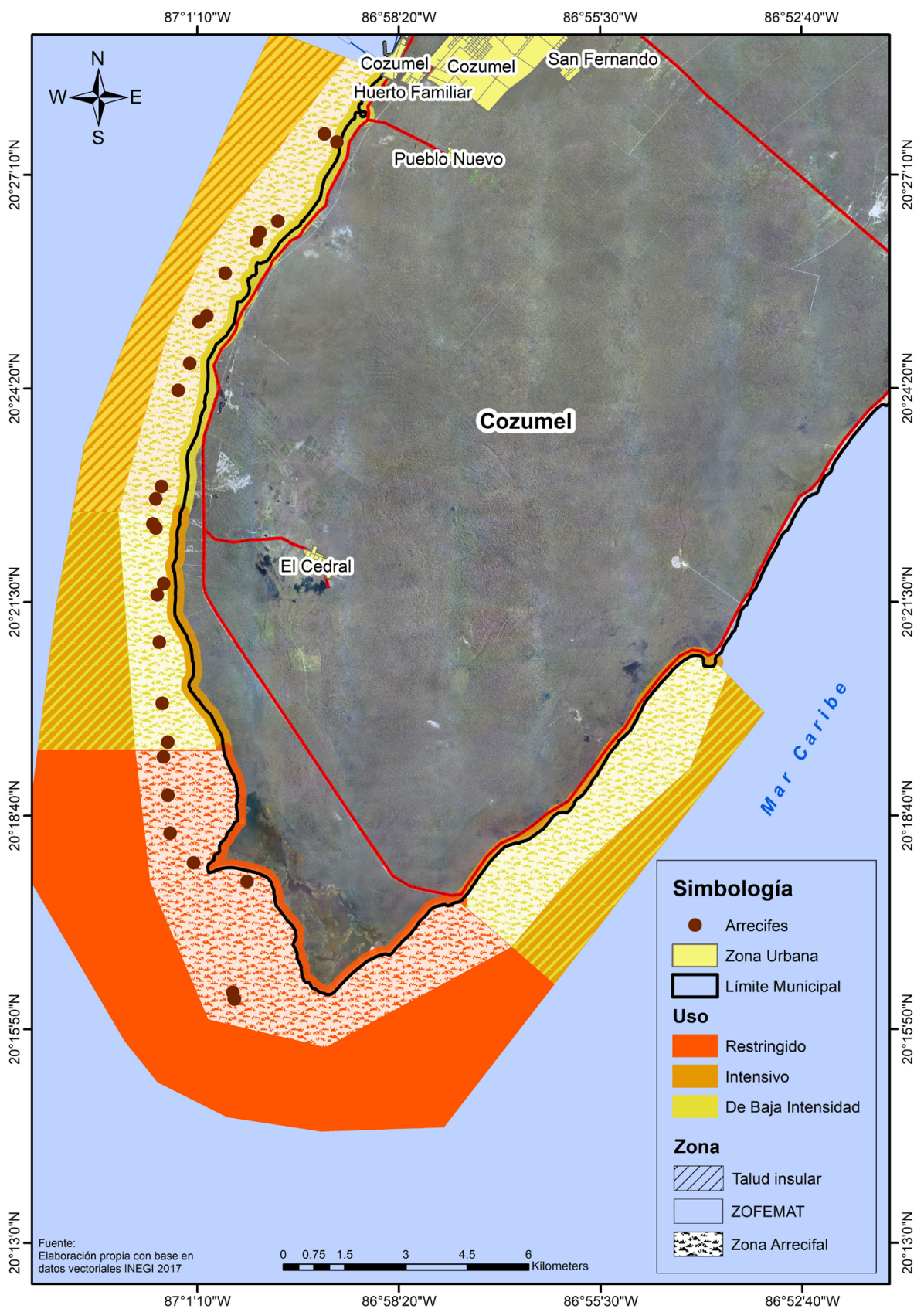

Fuente: Elaboración propia con base en el Programa de Manejo PNAC (1998); INEGI (2013). 
Lucinda Arroyo Arcos, Romano Gino Segrado Pavón, Oscar Frausto Martínez, Cristopher Arturo González Baca, Orlando Colín Olivares. Organización territorial y aprovechamiento turístico sustentable en el Parque Nacional Arrecifes de Cozumel, México

\section{Autorizaciones y oferta turística en el área de influencia}

Desde antes de la creación del PNAC, los residentes de Cozumel habían identificado al turismo marino como alternativa productiva y al año 2014 se ofrecía una amplia variedad de actividades turísticas y recreativas tanto en el área de influencia al ANP, como aquellas dentro del polígono, asociadas a 33 arrecifes coralinos en aguas relativamente someras y tranquilas, como por ejemplo buceo, pesca deportiva, esnórquel entre otras actividades como paseos en motos acuáticas, kayak, o distracción con juegos acuáticos o de playa. En el año 2014 se tenían registros de haber otorgado 425 autorizaciones y permisos (CONANP, 2014), con el siguiente detalle (Ver tabla 2).

Tabla 2. Permisos para prestar servicios en el PNAC

\begin{tabular}{|l|c|}
\hline \multicolumn{1}{|c|}{ Tipo de permiso } & Total \\
\hline Para prestar servicios turísticos con vehículo (embarcación menor o mayor) & 245 \\
\hline Para prestar servicios turísticos con vehículo (moto acuática) & 19 \\
\hline $\begin{array}{l}\text { Para prestar servicios turísticos con vehículos no motorizados (kayak, } \\
\text { velero, flotador, etc.) }\end{array}$ & 141 \\
\hline Para prestar servicios turísticos sin vehículo & 20 \\
\hline Total de permisos & 425 \\
\hline
\end{tabular}

Fuente: CONANP, 2014.

Las autorizaciones totalizan 3868 asientos, que representan la cantidad diaria de visitantes que podrían acceder al PNAC de forma regulada, aunque según comentarios de los permisionarios existen embarcaciones sin autorización que también prestan servicios similares de forma cotidiana (Talleres con permisionarios, 2014). Con relación al comentario anterior, según el estudio "Impacto de los visitantes en el Parque Nacional Arrecifes de Cozumel, derivado de las actividades turísticas: buceo autónomo y buceo libre" el Departamento de Monitoreo Biológico y Vinculación Académica (PNAC-CONANP, 2013), reportó que el 37\% de las embarcaciones evaluadas para buceo libre no estaban autorizadas para prestar dicho servicio, mientras que en el caso de las embarcaciones de buceo autónomo, el 7\% no estaba autorizadas.

En los recorridos de campo fue posible identificar en el área de Influencia a la zona protegida, servicios de usos turísticos, recursos o atractivos de uso público: cinco playas públicas, 15 clubes de playas, ocho restaurantes, 18 servicios de hospedaje entre hoteles, villas condominios o segundas residencias, una marina, tres muelles turísticos, 18 muelles de amarre, entre otros (Ver Ilustración 3). 
Lucinda Arroyo Arcos, Romano Gino Segrado Pavón, Oscar Frausto Martínez, Cristopher Arturo González Baca,

Orlando Colin Olivares. Territorial organization and sustainable tourist use in the Cozumel

Reefs National Park, México

Ilustración 3. Atractivos y oferta turística en el área de influencia del PNAC

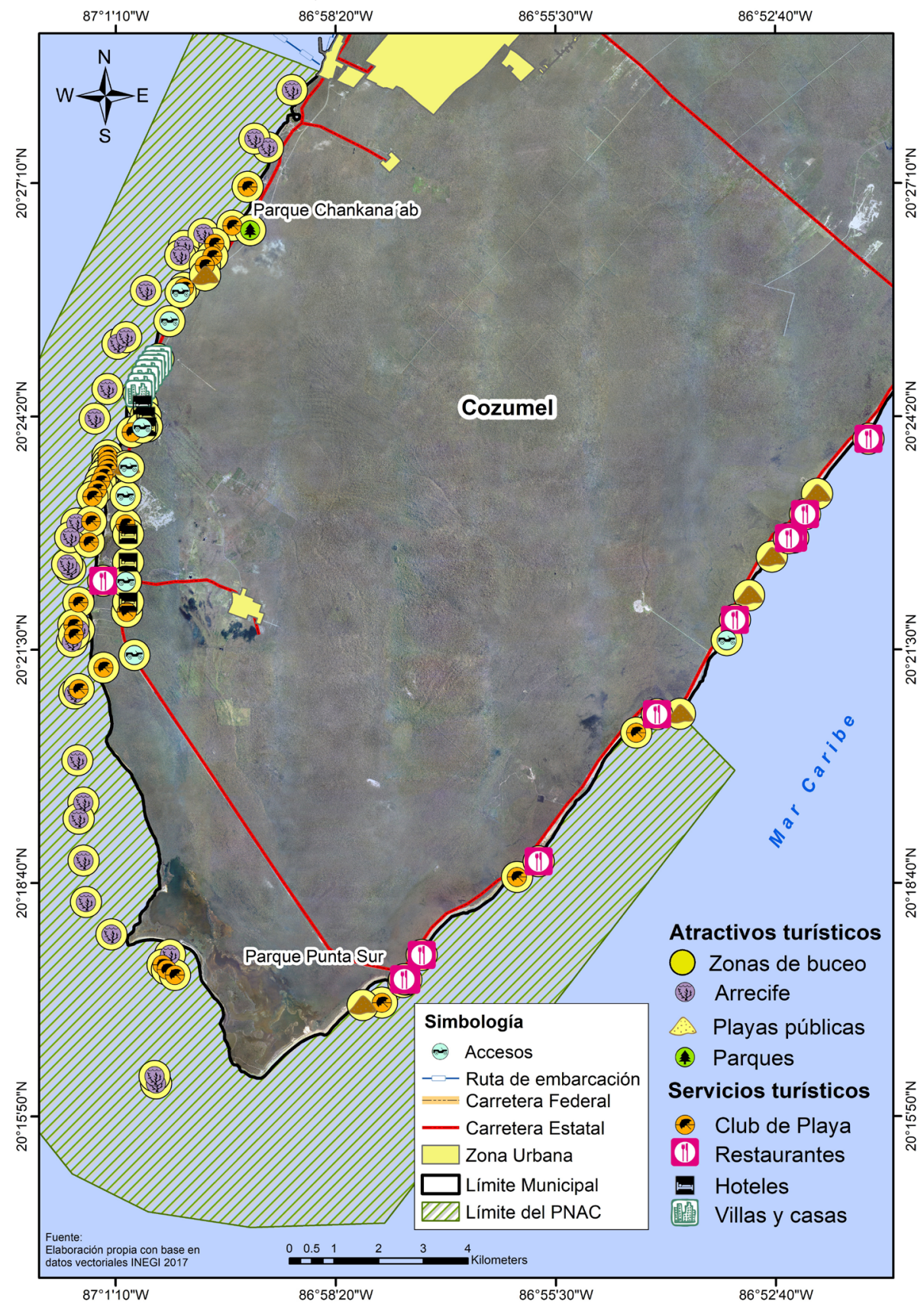

Fuente: Elaboración propia con base en el trabajo de campo, 2014. 
Lucinda Arroyo Arcos, Romano Gino Segrado Pavón, Oscar Frausto Martínez, Cristopher Arturo González Baca, Orlando Colín Olivares. Organización territorial y aprovechamiento turístico sustentable en el Parque Nacional Arrecifes de Cozumel, México

\section{FODA del Parque Nacional Arrecifes de Cozumel}

El análisis de Fortalezas, Oportunidades, Debilidades y Amenazas (FODA), se aplica para la toma de decisiones en organizaciones (Kangas, Kurtila, Kajanus y Kangas, 2003; Rauch, 2007). Está compuesto de afirmaciones con descripciones de factores internos y externos, que corresponde a un análisis subjetivo de una situación. En el ramo turístico, Wrigley y Gould (2002), refieren que el FODA se utiliza en la administración de ANP para fomentar la participación de la comunidad o para elaborar planes de desarrollo turístico (Ramos, Salazar y Gomes, 2000). Este tipo de análisis cualitativo no determina correctamente la importancia de los factores analizados o establece de forma adecuada las prioridades, pero proporcionan información útil para la gestión.

Para efectos del presente documento destacan los principales enunciados del análisis FODA (Entrevistas a informantes clave y talleres con permisionarios, 2014). En las Fortalezas, el paisaje arrecifal coralino se recalca por su carácter icónico en el Caribe Mexicano, el alto nivel de organización, así como la predisposición de los usuarios a colaborar en la conservación del ANP. Otras de las Fortalezas es la motivación de la comunidad por conservar y planificar el aprovechamiento.

Entre las Oportunidades sobresale el reconocimiento internacional obtenido por el ANP que ha favorecido el apoyo de organizaciones extranjeras con proyectos de conservación y capacitación, lo que favorecerá ampliamente las estrategias de manejo del PNAC. En las principales Debilidades resalta la baja credibilidad de algunos sectores sociales, el desconocimiento por parte de la comunidad acerca de la sub-zonificación turística, poca participación local y ausencia de un centro de interpretación ambiental. Con respecto a las Amenazas, es el crecimiento desorganizado de la actividad turística y el desarrollo de actividades humanas no compatibles en la zona de influencia del PNAC que ponen en riesgo la integridad de los recursos naturales del ANP. El entorno, con sus manifestaciones antrópicas negativas con base en el bajo nivel de educación y poca aplicación de sanciones podría ser la causa principal de afectación hacia los arrecifes.

\section{Aprovechamiento turístico en el PNAC}

La sub-zonificación secundaria es una estrategia congruente con la definida en el Programa de Manejo del Área Natural Protegida, que 
establece las sub-zonas donde resulta admisible el uso público y turísticorecreativo. Esta sub-zonificación secundaria permite ordenar la distribución de los visitantes y es un factor crítico para alcanzar la combinación adecuada entre concentración y dispersión. La definición o el establecimiento de límites de una sub-zona secundaria en el PNAC debe realizarse a manera de "unidades funcionales recreativas" que permitan asignaciones de intensidades de uso recreativo y faciliten el manejo de la actividad, considerando que en la mayoría de los casos el nodo central son los arrecifes. Con base en el diagnóstico se sugirieron las siguientes sub-zonas secundarias para el caso específico del PNAC, consistentes con su zonificación vigente según el Programa de Manejo (1998) y los criterios establecidos en la LGEEPA (Sub-zona de Preservación -ZP, Sub-zona de Aprovechamiento Sustentable de los Recursos Naturales -ZAS, Sub-zona de Uso Público -ZUP, Sub-zona de Uso Restringido -ZUR) y Sub-zona de Protección (ZPT) (Ver tabla 3).

Tabla 3. Intensidad de Uso

\begin{tabular}{|l|l|l|l|l|l|}
\hline \multicolumn{1}{|c|}{ Zona Recreativa } & Sin uso & Muy baja & \multicolumn{1}{c|}{ Baja } & Media & Alta \\
\hline ZP. Protección & & & $\mathrm{X}$ & & \\
\hline $\begin{array}{l}\text { ZAS. Aprovechamiento } \\
\text { Sustentable }\end{array}$ & & & & $\mathrm{X}$ & \\
\hline ZUP. Uso Público & & & & & $\mathrm{X}$ \\
\hline ZUR. Uso Restringido & & $\mathrm{X}$ & & & $\mathrm{X}$ \\
\hline ZPT. Preservación & & $\mathrm{X}$ & & & \\
\hline
\end{tabular}

Fuente: LGEEPA, Artículos 49 y 52 (Última Reforma DOF 21-05-2014).

El PNAC, es un espacio turístico que recibe diariamente a turistas y excursionistas de cruceros, lo que conlleva una fuerte presión al interior del sitio. Según datos de Comisión Nacional de Áreas Naturales Protegidas (CONANP, 2014), en 2011 pagaron su cuota de acceso o brazalete 289,084 personas, en 2012 fueron 299,395 visitantes y en 2013 el total fue de 291,802 turistas y 313, 421 en el año 2014, no se encontraron datos precisos del número de buceadores y esnorquelistas que visitan cada arrecife (CONANP, 2014). Los resultados de este documento son parte del diagnóstico realizado en el marco del proyecto Limite de Cambio Aceptable del PNAC, financiado por la Comisión Nacional de Áreas Naturales 
Lucinda Arroyo Arcos, Romano Gino Segrado Pavón, Oscar Frausto Martínez, Cristopher Arturo González Baca, Orlando Colín Olivares. Organización territorial y aprovechamiento turístico sustentable en el Parque Nacional

Arrecifes de Cozumel, México

Protegidas (CONANP) del municipio de Cozumel en Quintana Roo, México y el Programa para la Integración o Modificación de los Programas de Manejo de las Áreas Naturales Protegidas (ANP) Competencia de la Federación (PROMANP).

Para lograr un mejor aprovechamiento sustentable y cumplimiento de la imagen objetivo, se realizó una propuesta de unidades funcionales, los criterios fundamentales fueron las actividades turístico-recreativas que se desarrollan actualmente dentro del ANP en las principales zonas arrecifales que son los atractivos icónicos del área y su intensidad de uso. Con estos argumentos iniciales el espacio territorial que ocupa el PNAC, se ha sido dividido en tres unidades funcionales, de acuerdo con observaciones sobre su dinámica y funcionamiento (Ver tabla 4). Se destacan algunos accesos importantes en la ciudad de San Miguel de Cozumel y en la zona de influencia del PNAC. Asimismo, se pudo constatar que las zonas arrecifales de mayor concentración tanto de embarcaciones como personas que practican alguna actividad de buceo, esnórquel, y recorridos en lancha son: Arrecife Paraíso, Palancar Chankana'ab, Tunich, Tormentos, San Francisco, Santa Rosa, Yuncab, Colombia y el El cielo, las actividades actuales, horario, duración y público objetivo (Taller, con permisionarios y cooperativistas, Trabajo de campo, 2014).

Tabla 4. Unidades funcionales para actividades turístico-recreativas

\begin{tabular}{|c|c|c|c|c|c|}
\hline \multicolumn{6}{|c|}{ Unidad funcional 1, en operación: Zona arrecifal } \\
\hline Accesos & $\begin{array}{c}\text { Puntos de } \\
\text { concentración }\end{array}$ & $\begin{array}{c}\text { Actividades } \\
\text { actuales }\end{array}$ & Horario & Duración & $\begin{array}{l}\text { Público } \\
\text { objetivo }\end{array}$ \\
\hline $\begin{array}{l}\text { El acceso } \\
\text { principal es } \\
\text { la Caleta y } \\
\text { en menor } \\
\text { medida, } \\
\text { distintos } \\
\text { muelles } \\
\text { privados } \\
\text { sobre el } \\
\text { litoral } \\
\text { (ZOFEMAT) }\end{array}$ & $\begin{array}{l}\text { Arrecife Paraíso, } \\
\text { Palancar } \\
\text { Chankana'ab, } \\
\text { Tunich, } \\
\text { Tormentos, San } \\
\text { Francisco, Santa } \\
\text { Rosa, Yuncab, } \\
\text { Colombia y El } \\
\text { Cielo }\end{array}$ & $\begin{array}{l}\text { Recorridos } \\
\text { turísticos, } \\
\text { buceo, } \\
\text { esnórquel. }\end{array}$ & $\begin{array}{l}\text { Normal: } \\
\text { diurno. } \\
\text { Especial: } \\
\text { nocturno. }\end{array}$ & $\begin{array}{l}\text { Tiempos } \\
\text { variables, } \\
\text { promedio } \\
\text { estimado } \\
\text { de cuatro } \\
\text { horas. }\end{array}$ & $\begin{array}{l}\text { Personas } \\
\text { de } 20 \text { a } 60 \\
\text { años, aunque } \\
\text { otras edades } \\
\text { también son } \\
\text { demandantes. }\end{array}$ \\
\hline
\end{tabular}


Lucinda Arroyo Arcos, Romano Gino Segrado Pavón, Oscar Frausto Martínez, Cristopher Arturo González Baca, Orlando Colin Olivares. Territorial organization and sustainable tourist use in the Cozumel Reefs National Park, México

\begin{tabular}{|c|c|c|c|c|c|}
\hline \multicolumn{6}{|c|}{$\begin{array}{c}\text { Unidad funcional 2, en operación: Áreas de apoyo al servicio turístico, en litoral (Ubicadas } \\
\text { en la zona de influencia del PNAC) }\end{array}$} \\
\hline Accesos & $\begin{array}{c}\text { Puntos de } \\
\text { concentración }\end{array}$ & \begin{tabular}{|c|}
$\begin{array}{c}\text { Actividades } \\
\text { actuales }\end{array}$ \\
\end{tabular} & Horario & Duración & $\begin{array}{l}\text { Público } \\
\text { objetivo }\end{array}$ \\
\hline $\begin{array}{l}\text { Distintos } \\
\text { muelles } \\
\text { privados } \\
\text { sobre el } \\
\text { litoral } \\
\text { (ZOFEMAT), } \\
\text { Puerto de } \\
\text { Abrigo, } \\
\text { muelles de } \\
\text { Playa del } \\
\text { Carmen }\end{array}$ & $\begin{array}{l}\text { La Caleta, muelles } \\
\text { privados de } \\
\text { hoteles y tour } \\
\text { operadores. }\end{array}$ & $\begin{array}{l}\text { Servicios de } \\
\text { recepción, } \\
\text { embarque y } \\
\text { desembarque, } \\
\text { alimentos } \\
\text { y bebidas; } \\
\text { venta de } \\
\text { suvenir. }\end{array}$ & Diurno & \begin{tabular}{|l} 
Tiempo \\
variable, el \\
promedio \\
estimado es \\
una hora.
\end{tabular} & $\begin{array}{l}\text { Personas que } \\
\text { contratan } \\
\text { servicios } \\
\text { turístico- } \\
\text { recreativos al } \\
\text { arrecife. }\end{array}$ \\
\hline \multicolumn{6}{|c|}{$\begin{array}{l}\text { Unidad funcional 3, en gestación: Atractivos turísticos potenciales en litoral (Ubicas en la } \\
\text { zona de influencia del PNAC) }\end{array}$} \\
\hline Accesos & $\begin{array}{c}\text { Puntos de } \\
\text { concentración }\end{array}$ & $\begin{array}{l}\text { Actividades } \\
\text { actuales }\end{array}$ & Horario & Duración & $\begin{array}{l}\text { Público } \\
\text { objetivo }\end{array}$ \\
\hline $\begin{array}{l}\text { Zona oriental } \\
\text { de la isla }\end{array}$ & $\begin{array}{l}\text { Muelle fiscal, } \\
\text { muelles privados } \\
\text { de hoteles y tour } \\
\text { operadores. }\end{array}$ & $\begin{array}{l}\text { Conservación } \\
\text { participativa: } \\
\text { observación } \\
\text { de tortugas. }\end{array}$ & $\begin{array}{l}\text { Nocturno, } \\
\text { principalmente. }\end{array}$ & \begin{tabular}{|l} 
Tiempo \\
variable, el \\
promedio \\
estimado es \\
tres horas.
\end{tabular} & Todo público \\
\hline $\begin{array}{l}\text { Centro de } \\
\text { interpretación } \\
\text { arrecifal y } \\
\text { museo. }\end{array}$ & $\begin{array}{l}\text { Centro de } \\
\text { interpretación } \\
\text { arrecifal y museo. }\end{array}$ & & Diurno & $\begin{array}{l}\text { Tiempo } \\
\text { variable, el } \\
\text { promedio } \\
\text { estimado es } \\
\text { una hora. }\end{array}$ & Todo público. \\
\hline
\end{tabular}

Fuente: Talleres con permisionarios, 2014; observación de campo, 2014.

Dentro de la organización del territorio y la planificación turística, los nodos requieren trayectorias para crear una red apropiada y estable, con nodos de descanso y nodos de actividades recreativas adecuados para distintos tipos de usuarios. Debido a la característica esencial del PNAC, con un ambiente acuático, los arrecifes son los atractivos principales y los nodos centrales. Las visitas a los arrecifes del PNAC se realizan de forma parcial, ya que son visitados principalmente los arrecifes con mejores atributos paisajísticos. En el PNAC existen aproximadamente 33 sitios arrecifales (Ver tabla 5) a los que se puede acceder, sin embargo un turista visita un promedio de dos a tres arrecifes en un día común (Trabajo de campo, 2014). El aprovechamiento de los nodos turísticos arrecifales se realizan 
Lucinda Arroyo Arcos, Romano Gino Segrado Pavón, Oscar Frausto Martínez, Cristopher Arturo González Baca, Orlando Colín Olivares. Organización territorial y aprovechamiento turístico sustentable en el Parque Nacional Arrecifes de Cozumel, México

por principios de cercanías y oportunismo, combinados con criterios económicos de rentabilidad a muy corto plazo, lo que influye en los nodos y en las actividades, lo que dificulta el ordenamiento y distribución de las mismas en el ANP.

La Tabla 5 presenta los nodos de actividades del PNAC, con una clasificación en principales, primarios y secundarios, con criterios de espacialidad y concentración turística (García, 2003).

Tabla 5. Sitios arrecifales de Cozumel y Nodos del PNAC

\begin{tabular}{|l|l|l|l|l|l|l|}
\hline Orden & Atractivo/Nodo & Principal & Primario & Secundario & $\begin{array}{c}\text { Profundidad } \\
\text { media (*) en } \\
\text { pies }\end{array}$ & $\begin{array}{c}\text { Nivel de } \\
\text { experiencia } \\
\text { de buceo }\end{array}$ \\
\hline $\mathbf{1}$ & Colombia bajo & & X & & 25 a 40 & Intermedio \\
\hline $\mathbf{2}$ & $\begin{array}{l}\text { Colombia } \\
\text { profundo }\end{array}$ & & & X & 60 a 90 & Avanzado \\
\hline $\mathbf{3}$ & Chunchaka'ab & & & X & 60 a 80 & Avanzado \\
\hline $\mathbf{4}$ & $\begin{array}{l}\text { Maracaibo } \\
\text { profundo }\end{array}$ & & & X & 70 a 150 & Avanzado \\
\hline $\mathbf{5}$ & Punta Celarain & & & Esnórquel & 5 a 20 & Principiante \\
\hline $\mathbf{6}$ & Punta Sur bajo & & X & & 20 a 70 & Intermedio \\
\hline $\mathbf{7}$ & $\begin{array}{l}\text { Punta Sur } \\
\text { profundo }\end{array}$ & & & X & 40 a 120 & Avanzado \\
\hline $\mathbf{8}$ & Paso El Cedral & & & X & 30 a 60 & Intermedio \\
\hline $\mathbf{9}$ & $\begin{array}{l}\text { Paso El Cedral } \\
\text { profundo }\end{array}$ & & & X & 30 a 100 & Avanzado \\
\hline $\mathbf{1 0}$ & Punta Francesa & & X & & 30 a 60 & Intermedio \\
\hline $\mathbf{1 1}$ & $\begin{array}{l}\text { Palancar La } \\
\text { Herradura }\end{array}$ & X & & & 40 a 60 & Principiante \\
\hline $\mathbf{1 2}$ & Palancar profundo & & & X & 50 a 120 & Avanzado \\
\hline $\mathbf{1 3}$ & Palancar Ladrillos & & & X & 50 a 90 & Avanzado \\
\hline $\mathbf{1 4}$ & Palancar Jardines & & X & & 30 a 80 & Principiante \\
\hline $\mathbf{1 5}$ & $\begin{array}{l}\text { Palancar } \\
\text { Cuevones }\end{array}$ & & X & & 50 a 120 & Avanzado \\
\hline $\mathbf{1 6}$ & Punta Dalila & & & X & 30 a 60 & Principiante \\
\hline $\mathbf{1 7}$ & $\begin{array}{l}\text { Santa Rosa } \\
\text { profundo }\end{array}$ & & & X & 30 a 100 & Avanzado \\
\hline $\mathbf{1 8}$ & Cardona & & & X & 20 a 30 & Principiante \\
\hline $\mathbf{1 9}$ & $\begin{array}{l}\text { Pecio C-53 Cañón. } \\
\text { Ficotencatl }\end{array}$ & & & 50 a 70 & Avanzado \\
\hline $\mathbf{2 0}$ & $\begin{array}{l}\text { Chankana'ab } \\
\text { Bolones }\end{array}$ & $\mathrm{X}$ & & 40 a 70 & Principiante \\
\hline
\end{tabular}


Lucinda Arroyo Arcos, Romano Gino Segrado Pavón, Oscar Frausto Martínez, Cristopher Arturo González Baca, Orlando Colin Olivares. Territorial organization and sustainable tourist use in the Cozumel Reefs National Park, México

\begin{tabular}{|l|l|l|l|l|l|l|}
\hline Orden & Atractivo/Nodo & Principal & Primario & Secundario & $\begin{array}{c}\text { Profundidad } \\
\text { media (*) en } \\
\text { pies }\end{array}$ & $\begin{array}{c}\text { Nivel de } \\
\text { experiencia } \\
\text { de buceo }\end{array}$ \\
\hline $\mathbf{2 1}$ & $\begin{array}{l}\text { Chankana'ab } \\
\text { Cuevas }\end{array}$ & X & & & 35 a 40 & Principiante \\
\hline $\mathbf{2 2}$ & $\begin{array}{l}\text { Chankana'ab } \\
\text { bajos }\end{array}$ & X & & & 30 a 50 & Principiante \\
\hline $\mathbf{2 3}$ & Dzul - Ha & & & Esnórquel & 5 a 20 & Principiante \\
\hline $\mathbf{2 4}$ & Las Palmas & & & X & 40 a 70 & Principiante \\
\hline $\mathbf{2 5}$ & Paraíso & X & & & 25 a 45 & Principiante \\
\hline $\mathbf{2 6}$ & Punta Tunich bajo & & X & & 50 a 60 & Intermedio \\
\hline $\mathbf{2 7}$ & $\begin{array}{l}\text { Punta Tunich } \\
\text { profundo }\end{array}$ & & & X & 50 a 120 & Avanzado \\
\hline $\mathbf{2 8}$ & San Clemente & & & X & 30 a 40 & Principiante \\
\hline $\mathbf{2 9}$ & San Francisco & X & & & 45 a 120 & Intermedio \\
\hline $\mathbf{3 0}$ & Santa Rosa bajo & X & & & 50 a 120 & Intermedio \\
\hline $\mathbf{3 1}$ & Tormentos & & & X & 30 a 60 & Intermedio \\
\hline $\mathbf{3 2}$ & Yucab & & X & & 40 a 70 & Avanzado \\
\hline $\mathbf{3 3}$ & El Cielo & & & Esnórquel & 5 a 20 & Principiante \\
\hline & Caleta & NA & NA & NA & Fondeadero & \\
\hline & TOTAL & $\mathbf{7}$ & $\mathbf{7}$ & $\mathbf{1 9}$ & & \\
\hline
\end{tabular}

Fuente: Talleres con permisionarios, 2014. Observación de campo, 2014. Programa de Manejo PNAC, 1998. (*) Tres metros representa un aproximado a diez pies.

Para la organización territorial, el Programa de Manejo es una herramienta útil, que señala el tipo de intensidad de uso en el PNAC, con base en la propuesta, se observa una concentración en pocos nodos arrecifales categorizados como principales y dentro de la zona categorizada de uso restringido o de baja intensidad, los demás nodos arrecifales registran poca afluencia y se ubican en la zona categorizada de uso intensivo, lo que hace evidente que la realidad turística actual, es incompatible con la zonificación del Programa de Manejo del Parque vigente, al ser rebasado por las actividades turístico-recreativas que se practican en el área (Ver Ilustración 4). 
Lucinda Arroyo Arcos, Romano Gino Segrado Pavón, Oscar Frausto Martínez, Cristopher Arturo González Baca, Orlando Colín Olivares. Organización territorial y aprovechamiento turístico sustentable en el Parque Nacional Arrecifes de Cozumel, México

\section{Ilustración 4. Concentración en nodos arrecifales en el PNAC y uso} según zonificación

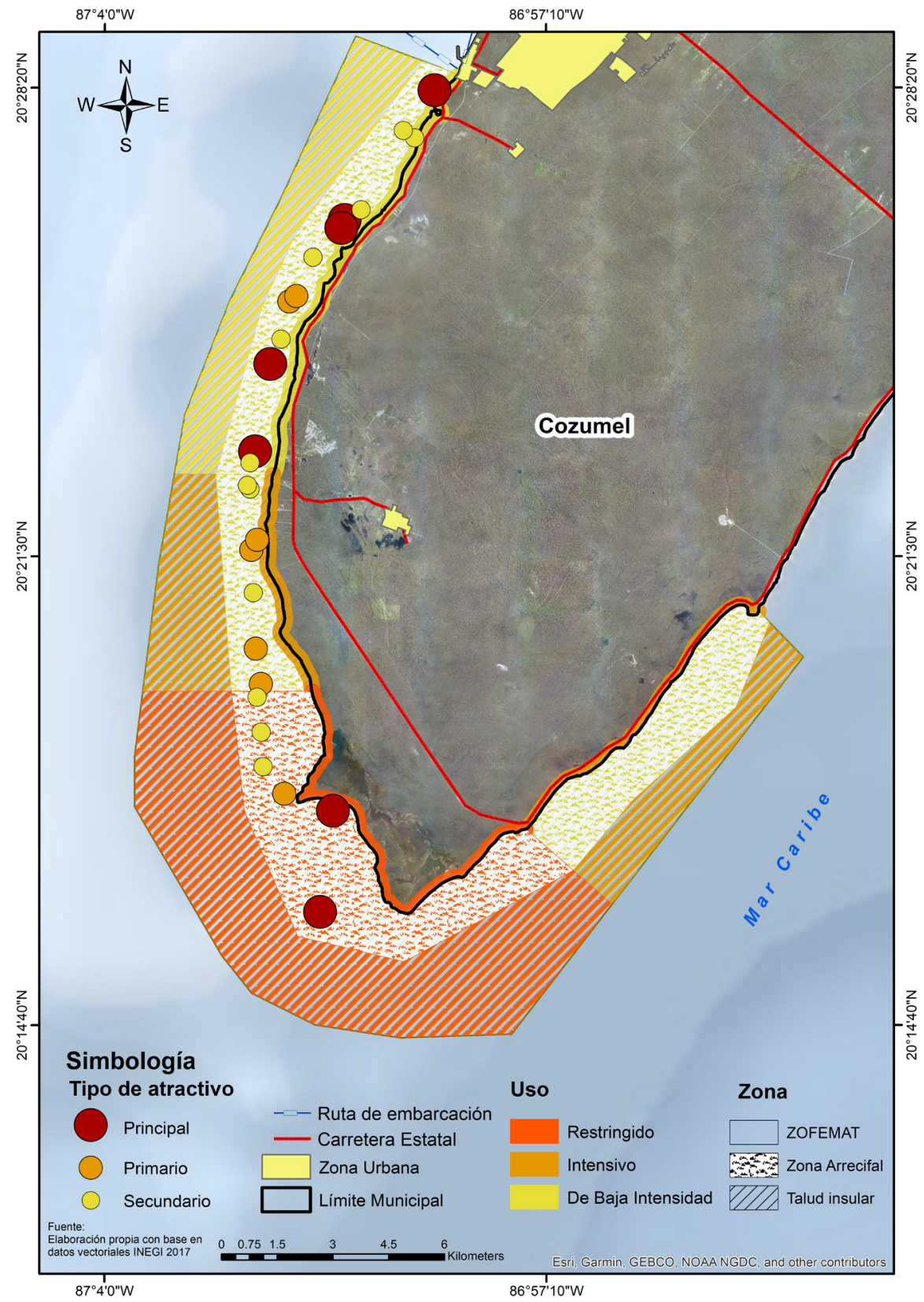

Fuente: Elaboración propia con base en el trabajo de campo, 2014 y Programa de Manejo, PNAC, 1998. 
Por lo que se observa en la ilustración 4 , la información debe ser útil para trabajar a corto plazo en la actualización del programa de manejo y establecer las medidas de control y monitoreo para la organización y planificación del territorio del parque, que vaya acorde con el objetivo por el que fue decretado, sin anteponer los intereses particulares que conlleva la actividad turística. Existe un disenso entre la comunidad cozumeleña, al observar que se está generando un impacto negativo por los permisos para la construcción y ampliación del muelle Puerta Maya, se alude a la pérdida de arrecifes y posible afectación al desarrollo natural de las especies de la zona, por lo que se debe profundizar la investigación científica sobre ese tema (Observación de campo, 2014 y Talleres con permisionarios y cooperativistas, 2014).

\section{Discusión y conclusión}

Cozumel se ha vuelto un destino masivo debido a los cruceros, y como señalan Pinassi y Ercolan (2017 y 2015); Hall (2013), las actividades de ocio se han convertido en una productora y consumidora de espacios, en el caso del PNAC, la orientación estratégica de aprovechamiento turístico sustentable debe considerar normas de restricción en las embarcaciones y los visitantes, que pueda ser monitoreado por un sistema Limite de Cambio Aceptable (LCA) y permita un impacto ambiental mínimo a los arrecifes, mientras se logra un alto nivel de contacto con la naturaleza y se realiza un impacto social positivo por medio de la generación de empleo para la comunidad local. En concordancia con López (2015); Vicencio y Bringas (2014), es necesario buscar la compatibilidad entre el turismo y el desarrollo sustentable que incluya a la comunidad.

La concentración de visitantes durante la visita al ANP en sitios o nodos específicos puede apoyar por una parte, a fortalecer el control y registro de los mismos, y por la otra, a promover la consciencia ambiental, informar e influir sobre las conductas requeridas por el usuario, previo a la visita al ANP.

Se observa una disminución de la vegetación en el área de influencia al PNAC, a raíz de creciente infraestructura urbana y turística en el litoral occidental de la isla, lo que ha modificado el paisaje y favorece efectos negativos indirectos en el PNAC (Entrevistas a informantes clave, 2014). Lo anterior es congruente con lo que menciona Briassoulis (2002), que al 
favorecer la transformación de espacios naturales a turísticos se potencializan los intereses opuestos al uso y conservación. Los espacios prístinos deberían, incluso en el entorno del PNAC, tener una jerarquía de conservación superior a su uso turístico y la infraestructura edilicia debe integrarse al paisaje circundante, en estricto cumplimiento de los objetivos y criterios del Programa de Ordenamiento Ecológico Territorial vigente en el municipio y el estado de Quintana Roo.

Dentro de la organización del territorio, se encontró como barrera legal, la falta de verificación sobre el uso del espacio litoral acorde a los límites establecidos en ordenamientos o concesiones por parte de ZOFEMAT, lo que ha permitido a los concesionarios extender la zona para uso exclusivo y omitir los pasos de servidumbres o accesos libres a las playas.

Otras posibles afectaciones sociales derivadas de la actividad turística en el área de influencia de PNAC, estará determinada por la habilitación de playas privadas, el crecimiento de la densidad poblacional en el litoral por medio de condominios, hoteles y otro tipo de alojamientos, que a su vez favorece la pérdida de vegetación, fragmentación del paisaje y la modificación del entorno por dragados, los permisos que se pudieran otorgar para la construcción de muelles y otra infraestructura de apoyo, por lo que debe apegarse a lo establecido en el Programa de Manejo del PNAC.

Por otra parte; el incremento poblacional, de las actividades agropecuarias de subsistencia, de las actividades turísticas y de infraestructura de apoyo al turismo masivo (en la Isla y la Riviera Maya), es posible que generen impactos indirectos negativos de largo plazo en los ecosistemas de las ANP. Actualmente este espacio protegido, está siendo aprovechado turísticamente, más no de forma sustentable, por lo que es necesario ampliar e incorporar estrategias interpretativas para favorecer la experiencia recreativa y la educación ambiental de turistas y población local. 


\section{Referencias}

Albet, A. (1993). "La nueva geografía regional o la construcción social de la región", Anales de la Geografía, núm. 13, pp.11-30.

Almirón, A. (2004). "Turismo y Espacio. Aportes para otra geografía del turismo", GEOUSP-Espaco e Tempo, núm. 16, pp. 166-180.

Briassoulis, H. (2002). "Sustainable tourism and the question of the commons". Annals of Tourism Research, núm. 29, 1065-1085.

CONANP, Comisión Nacional de Áreas Naturales Protegidas (2014), Presentación en Powerpoint sobre el Parque Nacional Arrecifes de Cozumel. Oficinas CONANP-Cozumel, Quintana Roo, México. Documento sin publicar.

CONANP. (2007). Programa de Turismo en Áreas Protegidas 2006-2012. Distrito Federal, México, México.

Delgadillo, J., Torres, F., y Gasca, J. (2001), El desarrollo regional de México en el vértice de dos milenios, Colección Textos Breves de Economía, Instituto de Investigaciones Económicas- UNAM, México.

Dilschneider, L. (2016). Propuesta de valorización del patrimonio ambiental para el desarrollo del ecoturismo en el Área Natural Protegida Caleta de los Loros-Pozo Salado-Punta Mejillón. Provincia de Río Negro. (Tesis de Grado) Recuperada de http://repositoriodigital. uns.edu.ar/handle/123456789/3323

Diario Oficial de la Federación (1996). DECRETO por el que se declara área natural protegida, con el carácter de Parque Marino Nacional, la zona conocida como Arrecifes de Cozumel. Diario Oficial de la Federación. 19 de julio de 1996, México.

Fenner, D. (1988). "Some leeward reefs and corals of Cozumel, México". Bulletin of Marine Science. vol. 42 (1), pp. 133-144.

García, M. (2003). Turismo y Conjuntos Monumentales: Capacidad de Acogida Turística y Gestión de Flujos de Visitantes. Fundación Cañada Blanch. Generalitat Valenciana. Ediciones Tirant lo Blanch, Valencia.

Gómez, F. (2005), “Aportaciones de la geografía al estudio científico del turismo", [en línea], Lurralde Investigación espacial, núm. 28. pp. 153-161. [consulta el 25 de abril, 2017], Disponible en: http://www. ingeba.org/lurralde/lurranet/lur28/28gomez/28gomez.htm

Hall, M. (2013). "Framing Tourism Geography: Notes from the Underground". Annals of Tourism Research, núm.43, pp.601-623. 
Lucinda Arroyo Arcos, Romano Gino Segrado Pavón, Oscar Frausto Martínez, Cristopher Arturo González Baca, Orlando Colín Olivares. Organización territorial y aprovechamiento turístico sustentable en el Parque Nacional

Arrecifes de Cozumel, México

Hiernaux, D y Lindón, A. (1993), "El Concepto del Espacio y el Análisis Regional”, SECUENCIA, núm. 3, pp. 89-111.

INE. Instituto Nacional de Ecología (1998). Programa de Manejo Parque Marino nacional Arrecifes de Cozumel, Quintana Roo, México.

Iradi, M. (2014) Las áreas naturales protegidas del sudoeste bonaerense: diseño de una ruta ecoturística (Tesis de Grado) Recuperada de http://repositoriodigital.uns.edu.ar/handle/123456789/3242

Kangas, J., Kurtila, M., Kajanus, M. y Kangas, A. (2003). "Evaluating the management strategies of a forestland estate-the S-O-S approach". Journal of Environmental Management, núm, 69, pp. 349-358.

Kostrowicki, J. (1986), Un concepto Clave: organización espacial, versión al español de Elizabeth Holt, Instituto de Geografía, UNAM, México.

Ley General de Equilibrio Ecológico y la Protección al Ambiente. (2012). Ley General de Equilibrio Ecológico y la Protección al Ambiente en materia de Áreas Naturales Protegidas. México: ISEF.

Ley General de Turismo. (2009). México: Diario Oficial de la Federación. Li, Ch. y Lian, L. (2012). "Theoretical research of the urban comprehensive carrying capacity in the epoch of urbanization". International Journal of Financial Research, núm. 3, 1.

López, A. (2015) “Turismo y desarrollo sustentable en áreas protegidas o sobre los "nuevos" contrasentidos para la producción y el marasmo en el ámbito rural", Desacatos, núm 47, pp. 36-53.

Miranda y Alvarado (2017) "Relaciones territoriales de la actividad turística en torno al Parque Nacional Rincón de la Vieja, Costa Rica", Revista Geográfica de América Central Nº Especial, pp. 185-207.

OMT (Organización Mundial del Turismo) (1981). Saturation of tourist destinations: Report of the secretary general. Madrid.

Pinassi, A. y Ercolan, P. (2017) “Turismo y Espacio Turístico: un análisis teórico conceptual desde la ciencia geográfica". Rev. Anais Bras. de Est. Tur./ ABET, Juiz de Fora, vol.7, núm.1, pp..42 - 61 .

Pinassi, A. y Ercolan, P. (2015) "Geografía del turismo: análisis de las publicaciones científicas en revistas turísticas. El caso de Argentina”, Cuadernos de Geografía - Revista Colombiana de Geografia, vol. 24, núm. 1, pp. 213-230.

Ramos, P., Salazar, A. y Gomes, J. (2000). "Trends in Portuguese Tourism: A Content Analysis of Association and Trade Representative 
Perspectives Intl. J. Contemporary Hospitality Management 12 (7): $409-416$.

Rauch, P. (2007). "SWOT analyses and SWOT strategy formulation for forest owner cooperations in Austria", Eur J Forest Res, vol. 126, pp. 413-420.

Reyes, H. (2007). Informe final del Proyecto DM007. Monitoreo complementarios de algas, invertebrados y peces en el Parque Nacional Arrecifes de Cozumel. Cozumel, Quintana Roo.

Sánchez Jasso, J. M., \& Cebrián Abellán, F. (2015). Turismo de naturaleza en Áreas Protegidas de México; una propuesta de conservación, aprovechamiento y desarrollo local en el Nevado de Toluca. Cuadernos de Turismo, 339-365.

Santos, M. (2000), La naturaleza del espacio. Técnica y tiempo. Razón y emoción, Ariel, Barcela.

Santos, M. (1990), Por una nueva Geografía, Espasa-Calpe, Madrid.

Segrado Pavón, R. G., Arroyo Arcos, L., Amador Soriano, K., Palma Polanco, M. (2015)."Hacia un modelo de aprovechamiento turístico sustentable en Áreas Naturales Protegidas: estudio de caso del Parque Natural Chankanaab de Cozumel, México”. Pasos. Revista de Turismo y Patrimonio Cultural, núm. 13, 26-42.

Segrado, R., Serrano, R., Isabel, J., Cruz, G., Balbuena, P. (2014). "Evaluación de dos métodos para el aprovechamiento turístico en Áreas Naturales Protegidas". Revista Iberoamericana de Economía Ecológica, núm. 23, 1-14.

Segrado, R., Arroyo L., y Amador K. (2010). "La zonificación y su aplicación en las áreas naturales protegidas de uso turístico de Quintana Roo”. E1 Periplo Sustentable, núm. 19. pp. 69-91. En http://www. uaemex.mx/plin/psus/periplo19/articulo_03.pdf

Segrado, R., y Arroyo, L. (2009) "El método de la Capacidad de Carga Turística Aplicado a la Medición de la Sustentabilidad de Cozumel, México". Revista TURyDES, Vol 2, No. 5. Internet: http://www.eumed.net/rev/turydes/05/sa.htm

SEGOB (2009): DOF: 13/02/2009 ACUERDO por el cual se establecen periodos de veda para la pesca comercial de caracol rosado o blanco (Strombus gigas) en aguas de jurisdicción federal correspondientes al litoral del Estado de Quintana Roo. Diario Oficial de la Federación, 
13 de febrero, 2009. Secretaría de Gobernación. Secretaria de Agricultura, Desarrollo Rural, Pesca y Alimentación, México.

SEMARNAT (2010) "NORMA Oficial Mexicana NOM-059-SEMARNAT-2010, Protección ambiental-Especies nativas de México de flora y fauna silvestres-Categorías de riesgo y especificaciones para su inclusión, exclusión o cambio-Lista de especies en riesgo". Diario oficial 30 de diciembre, 2010. Secretaria de Medio Ambiente y Recursos Naturales. México.

SECTUR (Secretaria de turismo de México) (2004) Turismo alternativo: una nueva forma de hacer turismo. Secretaria de turismo, México. En turismoalternativo@sectur.gob.mx www.sectur.gob.mx

Vera, F. (coord.), (1997), Análisis territorial del turismo. Barcelona: Ariel Vicencio, Y. y Bringas, N. (2014) "Conflictos entre la conservación y el turismo en áreas naturales protegidas: el buen vivir como aspiración para Bahía de los Ángeles”, Teoría y Praxis, núm. especial, pp. 49-73 Wrigley, M. y Gould, B. (2002). "Considering People, Adding Value, Maintaining Relevance: Strategies and tactics to Increase the Usage of Public Parks. J.” Leisure Property, vol. 2(2), 142 - 154. 
